\title{
The material-point method for granular materials
}

\author{
S.G. Bardenhagen ${ }^{\text {a }}$, J.U. Brackbill ${ }^{\text {b,* }}$, D. Sulsky ${ }^{\text {c }}$ \\ ${ }^{a}$ ESA-EA, MS P946 Los Alamos National Laboratory, Los Alamos, NM 87545, USA \\ b T-3, MS B216 Los Alamos National Laboratory, Los Alamos, NM 87545, USA \\ ${ }^{\mathrm{c}}$ Department of Mathematics and Statistics, University of New Mexico, Albuquerqe, NM 87131, USA
}

\begin{abstract}
A model for granular materials is presented that describes both the internal deformation of each granule and the interactions between grains. The model, which is based on the FLIP-material point, particle-in-cell method, solves continuum constitutive models for each grain. Interactions between grains are calculated with a contact algorithm that forbids interpenetration, but allows separation and sliding and rolling with friction. The particle-in-cell method eliminates the need for a separate contact detection step. The use of a common rest frame in the contact model yields a linear scaling of the computational cost with the number of grains. The properties of the model are illustrated by numerical solutions of sliding and rolling contacts, and for granular materials by a shear calculation. The results of numerical calculations demonstrate that contacts are modeled accurately for smooth granules whose shape is resolved by the computation mesh. (c) 2000 Elsevier Science S.A. All rights reserved.
\end{abstract}

\section{Introduction}

Granular materials are large conglomerations of discrete macroscopic particles, which may slide against one another but not penetrate [9]. Like a liquid, granular materials can flow to assume the shape of the container. Like a solid, they can support weight, but unlike a solid cannot support a tensile stress [11]. They are as common as sand on a beach or sugar on our breakfast cereal, yet they confound attempts to apply continuum models and pose subtle problems for statistical analysis [12].

Possibly, the most powerful way of modeling assemblies of grains is by numerical techniques. The flexibility of numerical modeling extends to loading configurations, grain sizes, size distributions and physical properties of the grains. Two approaches to modeling granular materials, relevant to the present study, are reviewed briefly.

Micromechanical modeling is developed to simulate the dynamic consolidation of metal powder in [24]. An Eulerian grain-level continuum model describes in detail the response of individual grains to an applied load. The approach is to reduce the scale of the region modeled to the point where only a few grains are considered. Conventional constitutive relations describe the material properties. Continuum mechanics equations are solved over the whole domain using initial and boundary conditions appropriate for the consolidation process. Finite difference approximations to the continuum equations are solved on an Eulerian mesh. Interface tracking resolves the material interfaces. The shear stress transfer across grain interfaces is assumed to be zero in the model; thus, friction between grains is neglected.

A mixture theory describing contact between grains has been developed by Benson [3] for an arbitrary Lagrangian-Eulerian micromechanical model. The model approximates stress equilibration mixture theory normal to the interface between two grains in contact, and mean strain rate theory tangential to the

\footnotetext{
${ }^{*}$ Corresponding author.
} 
interface. The contact mixture theory ensures the proper traction boundary conditions along the boundary. Its implementation requires the solution of simultaneous nonlinear algebraic equations in each cell occupied by more than one material. Benson notes there are errors in transport and the development of a boundary layer at the interface in the mixture model [3]. These appear to result from the inability to model sliding in a single-valued velocity field.

In contrast to micromechanical modeling, the distinct element method (DEM) computes the interactions of grains in a Lagrangian frame [6]. A granular material is composed of distinct grains which displace independently from one another and interact only at contact points. It is assumed the deformations of individual grains are small in comparison with the deformation of the granular assembly as a whole. The latter deformation is due primarily to the movements of grains as rigid bodies. Therefore, it is argued that precise modeling of particle deformation is not necessary to obtain a good approximation of the mechanical behavior.

In DEM, the equilibrium contact forces and the displacements of a stressed assembly of circular disks are found through a series of calculations tracing the movements of individual grains. The method of time integration is explicit, and the interaction of a large number of grains can be calculated at moderate cost. The interaction force between grains is in proportion to their overlap. Contact is detected when the distance between the centers of two grains is less than the sum of their radii. A Coulomb friction force can be included in the interaction, as well as more complex interactions to model material properties [15].

More recently, a DEM model for the interaction of convex polyhedral grains has been developed [7]. The contact model uses a common-plane to reduce the number of tests to detect contact. For example, tests for contact between two cubes are reduced from 240 to 16. The common-plane, which bisects the space between grains, is constructed by maximizing the gap between the common-plane and the closest vertex. The normal to the contact plane defines the interface normal, and all interactions between grains are calculated with reference to the common-plane. The complexity of this and other contact models for rigid bodies is reviewed in [1].

We are interested in simulating systems on the scale of many grains. Our goal is not only to allow each grain to deform according to continuum constitutive models, but also to describe the interactions between grains, including separation, sliding, and rolling. Our approach combines features of the micromechanical modeling approach, as described by Williamson [24], and the distinct element method [6]. In such a setting, the constitutive model is comparatively well defined, as are the interactions among the grains. The ultimate goals of simulations on this scale are to understand under what circumstances homogenized constitutive theories of granular materials are valid, and to suggest new theories based on observed behavior. In the process, fluctuations that may preclude application of such theories can be identified and studied.

Numerical simulation of granular flow provides many challenges. Since each grain is modeled as a separate body, an efficient technique for identifying contact between bodies is essential. Large deformations can also present difficulties associated with mesh distortion and implementation of path-dependent constitutive models. This paper describes the material-point method (MPM) for solving problems in this class. MPM is a particle-in-cell code based on the implicit, hydrodynamics code FLIP [4,5]. The method has been applied to impact and penetration problems, wave propagation and to manufacturing problems [18-22]. No slip contact between grains is contained in the basic algorithm at no additional cost. In this paper, frictional slip is added with an algorithm that is linear in the number of grains. The algorithm is illustrated on numerical solutions of sliding and rolling of a grain, and the shear deformation of a few hundred grains.

\section{The material-point algorithm}

The particle-in-cell method, FLIP, combines the strengths of Eulerian and Lagrangian descriptions of the material. The Lagrangian description is provided by discretizing each body by a collection of material points, and the Eulerian description is based on a background computational mesh. The material-point method extends these capabilities to materials modeling. Information carried by the material points is projected on to the background mesh where equations of motion are solved. The mesh solution is then used to update the material points. In Sulsky et al. [18,19], a weak formulation of the MPM algorithm for solid mechanics is given and the method is framed in terms of finite elements. The momentum equation 


$$
\rho \frac{\mathrm{d} \mathbf{v}}{\mathrm{d} t}=\nabla \cdot \boldsymbol{\sigma}
$$

where $\rho$ is the mass density, $\mathbf{v}$ the velocity and $\boldsymbol{\sigma}$ is the stress tensor, is solved in a Lagrangian frame on a finite element mesh. The time derivative that appears in Eq. (1) is the material derivative

$$
\frac{\mathrm{d}}{\mathrm{d} t}=\frac{\partial}{\partial t}+\mathbf{v} \cdot \nabla
$$

The Lagrangian formulation means that the acceleration does not contain the convection term which can cause significant numerical error in purely Eulerian approaches. During this Lagrangian phase of the calculation, each element is assumed to deform in the flow of material so that points in the interior of the element move in proportion to the motion of the nodes. That is, given the velocity at the nodes determined from Eq. (1), element shape functions are used to map the nodal velocity continuously to the interior of the element. The positions of the material points are then updated by moving them in this single-valued, continuous velocity field. Similarly, the velocity of a material point is updated by mapping the nodal accelerations to the material point position. Because the velocity field is single-valued, interpenetration of material is precluded, and also no-slip contact between impinging bodies is automatic.

With this method, the finite element mesh does not conform to the boundary of each grain being modeled. Instead, a computational domain is constructed in a convenient manner to cover the potential domain for the boundary-value problem being solved. Then each grain is defined by a collection of material points. As material points move, they transport material properties assigned to them without error. In FLIP, the material points carry enough information to reconstruct the solution; therefore one can choose whether to continue the calculation in the Lagrangian frame or map information from the material points to another grid. This feature avoids mesh tangling which can occur in a purely Lagrangian calculation under large strains, and allows one to choose the grid for computational convenience.

To describe the numerical algorithm in more detail, a calculation is broken up into discrete time steps of size $\Delta t$, and the solution is computed at discrete times, $t^{n}, n=0,1, \ldots$ At each time step, $\Delta t=t^{n+1}-t^{n}$, the mapping from material points to a grid provides the initial data for the solution to Eq. (1). Since there are generally more material points than grid points, the nodal velocities at the beginning of each time step are determined from the material-point velocities using a weighted least squares approach. The weighting is the mass of the material point. The result is the following equation which must be solved for the nodal velocities, $\mathbf{v}_{j}^{n}$,

$$
\sum_{j} m_{i j}^{n} \mathbf{v}_{j}^{n}=\sum_{p} m_{p} \mathbf{v}_{p}^{n} N_{i}\left(\mathbf{x}_{p}^{n}\right)
$$

In this equation, $m_{p}$ is the material-point mass, $\mathbf{v}_{p}^{n}$ the velocity of the material point at time $t^{n}, \mathbf{x}_{p}^{n}$ the position of the material point at time $t^{n}$, and $N_{i}$ is the element shape function associated with node $i$. The consistent mass matrix, $m_{i j}^{n}$, is

$$
m_{i j}^{n}=\sum_{p} m_{p} N_{i}\left(\mathbf{x}_{p}^{n}\right) N_{j}\left(\mathbf{x}_{p}^{n}\right)
$$

In practice, we generally replace $m_{i j}^{n}$ with a lumped, diagonal mass matrix so that Eq. (3) becomes

$$
m_{i}^{n} \mathbf{v}_{i}^{n}=\sum_{p} m_{p} \mathbf{v}_{p}^{n} N_{i}\left(\mathbf{x}_{p}^{n}\right)
$$

where the lumped masses are

$$
m_{i}^{n}=\sum_{p} m_{p} N_{i}\left(\mathbf{x}_{p}^{n}\right)
$$

The shape functions are formed from the tensor product of linear b-splines [8] for the simulations in this paper. 
For a method that is explicit in time, the right-hand side of Eq. (1) is also computed using data from the material points. For fluids or elastic solids, one can form gradients of the nodal velocity or displacement to obtain strain rate or strain for an element and then apply constitutive equations element-by-element to compute stress. The internal forces then come from taking the divergence of the element stresses. This is the approach used in FLIP. For history-dependent materials, it has been convenient to carry the deformation gradient and stress, as well as history variables along with the material points in the Lagrangian frame. It is this change that we call MPM. In its simplest form, strain increments are obtained from gradients of the nodal velocities evaluated at the material point positions. Then, given a strain increment at a material point, along with current values of history variables and material parameters, standard routines are used to evaluate the stress increment and update history variables. The internal forces at the nodes are then calculated directly from the stress at the material points. This way, each material point has its own clearly defined history and there is no need to try to map this history to the elements. If $\boldsymbol{\sigma}_{p}^{n}$ is the stress at the material point $p$ at time $t^{n}$ and $\mathbf{G}_{p i}^{n}$ represents the gradient of the nodal basis function associated with node $i$, evaluated at $\mathbf{x}_{p}^{n}$, then the internal force at the nodes is

$$
\mathbf{f}_{i}^{\mathrm{int}}=-\sum_{p} \mathbf{G}_{p i}^{n} m_{p} \boldsymbol{\sigma}_{p}^{n} / \rho_{p}
$$

where

$$
\mathbf{G}_{p i}^{n}=\left.\nabla N_{i}\right|_{\mathbf{x}_{p}} .
$$

To summarize the algorithm, the steps are:

1. Given the particle mass, $m_{p}$, position, $\mathbf{x}_{p}^{n}$, velocity, $\mathbf{v}_{p}^{n}$, density, $\rho_{p}$ and stress, $\boldsymbol{\sigma}_{p}$, form the lumped mass matrix (Eq. (6)), solve for the nodal velocity. (Eq. (5)) and form the internal force (Eq. (7)).

2. Solve the momentum equations for the nodal accelerations and velocities in a Lagrangian frame:

$$
m_{i}^{n}\left[\mathbf{v}_{i}^{n+1}-\mathbf{v}_{i}^{n}\right]=\Delta t \mathbf{f}_{i}^{\text {int }} .
$$

3. Update the solution at the material points by mapping the nodal values using the element shape functions. Positions and velocities are updated according to,

$$
\mathbf{x}_{p}^{n+1}=\mathbf{x}_{p}^{n}+\Delta t \sum_{i} \mathbf{v}_{i}^{n+1} N_{i}\left(\mathbf{x}_{p}^{n}\right)
$$

and

$$
\mathbf{v}_{p}^{n+1}=\mathbf{v}_{p}^{n}+\sum_{i}\left[\mathbf{v}_{i}^{n+1}-\mathbf{v}_{i}^{n}\right] N_{i}\left(\mathbf{x}_{p}^{n}\right) .
$$

Also update the deformation gradient for each particle, compute the strain using an appropriate strain measure and solve constitutive equations to update the stress, $\boldsymbol{\sigma}_{p}$.

4. Define a new finite element mesh, if necessary, and return to step 1 to begin a new time step.

\section{The contact algorithm}

The preceding algorithm is intended to solve for motions and deformations of the grains moving in the single-valued velocity field $\mathbf{v}_{i}$ defined at nodes $i=1, \ldots, N_{n}$. This velocity field, defined on the computational mesh, arises from integrating forward in time the field interpolated from all particles. Since this field is determined using mass weighting given in Eq. (5), we call this the center-of-mass velocity field. A similar procedure where loops range over only the material prints making up one grain at a time, would solve the equations of motion for each grain, ignoring the presence of the others. In that case, each node has a mesh velocity $\mathbf{v}_{i}^{g}$ associated with it where $g$ ranges from one to the total number of grains, $N_{g}$, and $i$ ranges over the nodes $i=1, \ldots, N_{n}$. Of course, since the shape functions have compact support, only those nodes in the vicinity of the grain will have a meaningful velocity and the grain velocity at other nodes will be zero. 
Obviously, the aggregate grain motion cannot be determined solely from consideration of each individual grain in isolation. The novelty here is that contacts between grains are handled by comparing the fields $\mathbf{v}_{i}^{g}$ to the single, center-of-mass field $\mathbf{v}_{i}$. The resulting algorithm is linear in the number of grains and requires no iteration.

Normal and tangential kinematic constraints on the velocity are handled separately. Suppose that the boundary of each grain has been identified and the unit outward normal $\mathbf{n}_{i}^{g}$ is known at grid points along the boundary. The exact computation of the normal will be given later. If one grain is isolated from the rest, not in contact with others, then the two fields $\mathbf{v}_{i}^{g}$ and $\mathbf{v}_{i}$ will be identical in the neighborhood of that grain. It is only as the grain approaches others that the two fields will differ. In fact, we define contact precisely when these fields differ. Constraints on the grain motion are necessary only when grains are approaching; given by the condition,

$$
\left(\mathbf{v}_{i}^{g}-\mathbf{v}_{i}\right) \cdot \mathbf{n}_{i}^{g}>0 .
$$

Eq. (12) is satisfied when the grain velocity is overtaking the center-of-mass velocity along the normal to the surface. Once this condition is satisfied, adjust the grain velocity to a new value $\tilde{\mathbf{v}}_{i}^{g}$ so that

$$
\tilde{\mathbf{v}}_{i}^{g} \cdot \mathbf{n}_{i}^{g}=\mathbf{v}_{i} \cdot \mathbf{n}_{i}^{g}
$$

holds. That is, the normal component of the grain velocity is set equal to the normal component of the center-of-mass velocity, or

$$
\tilde{\mathbf{v}}_{i}^{g}=\mathbf{v}_{i}^{g}-\left[\left(\mathbf{v}_{i}^{g}-\mathbf{v}_{i}\right) \cdot \mathbf{n}_{i}^{g}\right] \mathbf{n}_{i}^{g} .
$$

We know that the center-of-mass velocity does not allow interpenetration of the grains, so this choice is natural. Note that the inequality in (12) allows grains to separate freely without constraints.

The constraint Eq. (13) is equivalent to applying a normal force, $\mathbf{f}_{n, i}^{g}$, to the grain of magnitude

$$
f_{n, i}^{g}=-m_{i}^{g}\left[\left(\mathbf{v}_{i}^{g}-\mathbf{v}_{i}\right) \cdot \mathbf{n}_{i}^{g}\right] / \Delta t=m_{i}^{g}\left[\left(\tilde{\mathbf{v}}_{i}^{g}-\mathbf{v}_{i}^{g}\right) \cdot \mathbf{n}_{i}^{g}\right] / \Delta t .
$$

The mass of the grain at node $i, m_{i}^{g}$, is defined like the nodal mass above (6), except that only the particles making up grain $g$ are used

$$
m_{i}^{g}=\sum_{p=1}^{N_{p}^{g}} m_{p} N_{i}\left(x_{p}\right) .
$$

The upper limit on the sum, $N_{p}^{g}$, is the number of particles making up grain $g$.

If there is no friction between the grains then the above adjustment of the normal component of velocity is all that is required, and the tangential component of the grain velocity is unconstrained. On the other hand, frictional slip is accomplished by also adjusting the tangential component. To apply Coulomb friction, first calculate the force necessary to cause the grains to stick completely. Again, the comparison of the grain velocity to the center-of-mass velocity field provides exactly the correct constraint for no-slip contact. The relative tangential velocity is

$$
\left(\mathbf{v}_{i}^{g}-\mathbf{v}_{i}\right)-\left[\left(\mathbf{v}_{i}^{g}-\mathbf{v}_{i}\right) \cdot \mathbf{n}_{i}^{g}\right] \mathbf{n}_{i}^{g}=\mathbf{n}_{i}^{g} \times\left[\left(\mathbf{v}_{i}^{g}-\mathbf{v}_{i}\right) \times \mathbf{n}_{i}^{g}\right] .
$$

The constraining tangential force $\mathbf{f}_{\text {stick }, i}^{g}$ is

$$
\mathbf{f}_{\text {stick }, i}^{g}=-m_{i}^{g} \mathbf{n}_{i}^{g} \times\left[\left(\mathbf{v}_{i}^{g}-\mathbf{v}_{i}\right) \times \mathbf{n}_{i}^{g}\right] / \Delta t .
$$

The friction force equals the sticking force if the magnitude of the sticking force is small. That is, friction just balances the tangential force and prevents relative tangential motion, when the magnitude of the tangential force is small. For larger tangential forces, the magnitude of the friction force is proportional (with proportionality constant $\mu$ ) to the magnitude of the normal force and independent of the contact area. Limiting the frictional force to have magnitude less than the sticking force allows tangential slip between the contacting grains since the applied frictional force is not sufficient to prevent relative tangential motion. 
The constant $\mu$ is called the coefficient of friction. The direction of the frictional force is chosen as in (18) to oppose relative motion. Putting these requirements together yields,

$$
\mathbf{f}_{\text {fric }, i}^{g}=\frac{\mathbf{f}_{\text {stick }, i}^{g}}{\left|\mathbf{f}_{\text {stick }, i}^{g}\right|} \min \left(\mu\left|\mathbf{f}_{n, i}^{g}\right|,\left|\mathbf{f}_{\text {stick }, i}^{g}\right|\right) .
$$

Using (15) and (18), the frictional force can also be written

$$
\mathbf{f}_{\mathrm{fric}, i}^{g}=-\frac{m_{i}^{g}}{\Delta t} \frac{\mathbf{n}_{i}^{g} \times\left[\left(\mathbf{v}_{i}^{g}-\mathbf{v}_{i}\right) \times \mathbf{n}_{i}^{g}\right]}{\left|\left(\mathbf{v}_{i}^{g}-\mathbf{v}_{i}\right) \times \mathbf{n}_{i}^{g}\right|} \mu^{\prime}\left[\left(\mathbf{v}_{i}^{g}-\mathbf{v}_{i}\right) \cdot \mathbf{n}_{i}^{g}\right]=-\frac{m_{i}^{g}}{\Delta t} \mu^{\prime}\left[\left(\mathbf{v}_{i}^{g}-\mathbf{v}_{i}\right) \cdot \mathbf{n}_{i}^{g}\right]\left(\mathbf{n}_{i}^{g} \times \hat{\boldsymbol{\omega}}\right),
$$

where

$$
\mu^{\prime}=\min \left(\mu, \frac{\left|\left(\mathbf{v}_{i}^{g}-\mathbf{v}_{i}\right) \times \mathbf{n}_{i}^{g}\right|}{\left(\mathbf{v}_{i}^{g}-\mathbf{v}_{i}\right) \cdot \mathbf{n}_{i}^{g}}\right)
$$

and the unit vector $\hat{\boldsymbol{\omega}}$ is

$$
\hat{\boldsymbol{\omega}}=\frac{\left(\mathbf{v}_{i}^{g}-\mathbf{v}_{i}\right) \times \mathbf{n}_{i}^{g}}{\left|\left(\mathbf{v}_{i}^{g}-\mathbf{v}_{i}\right) \times \mathbf{n}_{i}^{g}\right|} .
$$

We see that the frictional force is in the tangential direction, $\mathbf{n}_{i}^{g} \times \hat{\boldsymbol{\omega}}$, and the magnitude is $\mu^{\prime}$ times the magnitude of the normal force.

Imposing the frictional constraint force (20) and the normal constraint force (15) leads to an altered grain velocity, $\tilde{\mathbf{v}}_{i}^{g}$ which is

$$
\tilde{\mathbf{v}}_{i}^{g}=\mathbf{v}_{i}^{g}-\left[\left(\mathbf{v}_{i}^{g}-\mathbf{v}_{i}\right) \cdot \mathbf{n}_{i}^{g}\right]\left(\mathbf{n}_{i}^{g}+\mu^{\prime} \mathbf{n}_{i}^{g} \times \hat{\boldsymbol{\omega}}\right) .
$$

It is this condition that gives the complete kinematical constraint required to ensure no normal penetration and sliding friction.

A value for the normal at nodes of the computational mesh for each grain, $\mathbf{n}_{i}^{g}$, is still needed to complete the description of the contact algorithm. For each grain, the particle mass is interpolated to element centers, $\mathbf{x}_{e}$ and divided by the element volume, $\Omega_{e}$, to obtain a density,

$$
\rho_{e}^{g}=\sum_{p=1}^{N_{i}^{g}} m_{p}^{g} S^{(2)}\left(\mathbf{x}_{e}-\mathbf{x}_{p}\right) / \Omega_{e} .
$$

The shape function, $S^{(2)}$, is constructed from the tensor product of quadratic b-splines [8,17]. The gradient of $\rho_{e}^{g}$ evaluated at the nodes of the computational mesh provides the normal direction at the surface of each grain. The interaction between grains is therefore not along a common normal, which results in small errors in momentum conservation.

As described, the contact algorithm applies a model for dry, sliding friction. In some simulations, kinetic friction may also play a role. In this case, the friction coefficient is typically reduced once the grains are in relative motion. In some circumstances, rolling friction might be more appropriate than sliding friction. A rolling friction model also tends to reduce the coefficient of friction. As a first approximation, the above sliding friction algorithm is felt to be reasonable, particularly since not much is known experimentally about the true magnitude of the frictional forces.

The key to the efficiency of the friction algorithm is that we do not compare the grain velocities $\mathbf{v}_{i}^{g}$ pairwise, rather we compare each $\mathbf{v}_{i}^{g}$ to the common center-of-mass field $\mathbf{v}_{i}$. Therefore, the adjustment of $\mathbf{v}_{i}^{g}$ for all $g$ is done in one sweep through the grains without any iteration. The resulting algorithm is linear in the number of grains.

\section{Numerical simulations}

Numerical simulations presented in this section are carried out in two dimensions. The first set of simulations involves a disk rolling on an inclined plane and is meant as a simple illustration and validation 
of the friction algorithm. A more stringent test of the contact algorithm comes in the second simulation consisting of a few hundred polydisperse grains undergoing a large shear deformation.

\subsection{Inclined plane simulation}

Fig. 1 shows the geometry for a computation with a cylinder on an inclined plane. In this example, a grain interacts with the boundary of the computational domain, where friction is applied. The plane is inclined at an angle $\theta$ from the horizontal in Fig. 1a, while gravity g points vertically downward. If the disk is rigid, its center-of-mass velocity is directed tangent to the surface of the inclined plane. If this direction is called the $x$-direction and the $y$-direction is orthogonal, then Fig. $1 \mathrm{~b}$ indicates the orientation for the numerical simulation. The inclined plane is aligned with the boundary of the computational mesh and gravity makes an angle $\theta$ to the vertical.

A rigid disk on an inclined surface will roll, and either stick or slip at the point of contact depending on the angle of inclination and friction coefficient. Specifically, if $\tan \theta>3 \mu$, where $\mu$ is the coefficient of friction, the disk will roll and slip; otherwise the disk will roll without slipping. For an initially stationary, rigid disk, the $x$-component of the center-of-mass position as a function of time, $x_{\mathrm{cm}}(t)$, is given by

$$
x_{\mathrm{cm}}(t)= \begin{cases}x_{0}+\frac{1}{2}|\mathbf{g}| t^{2}(\sin \theta-\mu \cos \theta), & \tan \theta>3 \mu(\text { slip }), \\ x_{0}+\frac{1}{3}|\mathbf{g}| t^{2} \sin \theta, & \tan \theta \leqslant 3 \mu(\text { stick }) .\end{cases}
$$

In this formula, $x_{0}$ is the $x$-component of the initial center-of-mass position, and $|\mathbf{g}|$ is the magnitude of the gravitational acceleration.

Simulations are performed with a disk that has radius $R=50 \mathrm{~cm}$, and gravitational acceleration with magnitude $9.8 \mathrm{~m} / \mathrm{s}^{2}$. The computational mesh has square elements with side length $25 \mathrm{~cm}=0.5 R$, so there are only four computational elements across the diameter of the disk and 9 material points per element. The first test of the friction algorithm involves varying the coefficient of friction $\mu$. Fig. 2 shows the centerof-mass position of the disk as a function of time for two values of the friction coefficient, $\mu=0.3$ and 0.9 , and the angle of inclination fixed at $\theta=\pi / 3$. The simulation involves a compliant, linear-elastic, deformable disk, with shear modulus $2.5 \mathrm{MPa}$, bulk modulus $10 \mathrm{MPa}$, and density $3000 \mathrm{~kg} / \mathrm{m}^{3}$. For the material points

(a)

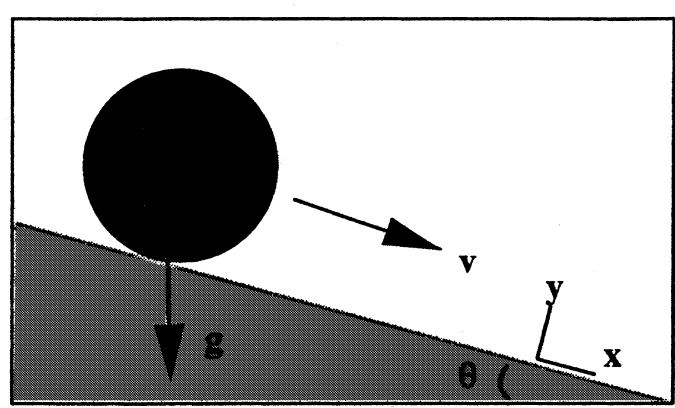

(b)

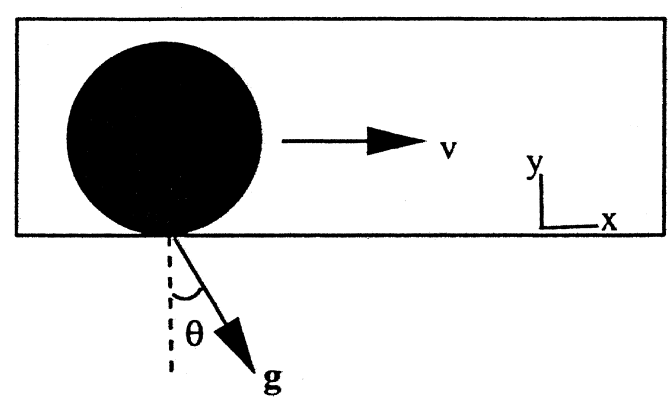

Fig. 1. Geometry for simulations of a disk on an inclined plane. 


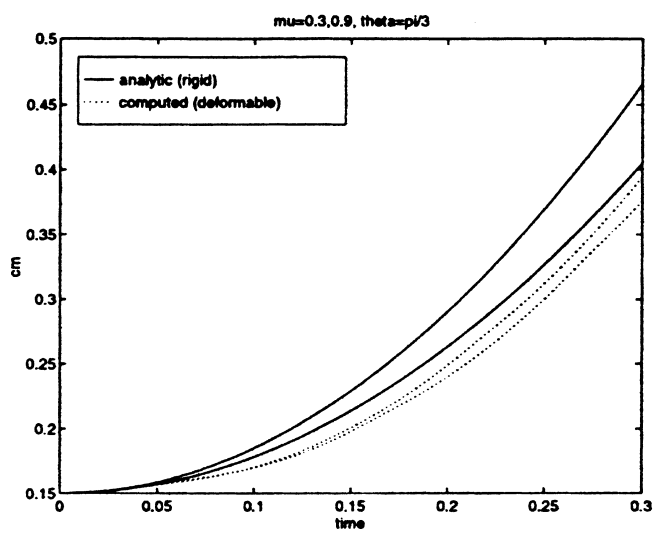

Fig. 2. Center-of-mass position ( $x$-component) for a deformable disk as a function of time (dotted lines) for simulations with friction coefficients $\mu=0.3$ and 0.9 , and angle of inclination $\theta=\pi / 3$. The analytical solution for a rigid disk (solid lines) is also shown for comparison. In both the computed and analytical solutions, the curves for $\mu=0.3$ lie above the curves for $\mu=0.9$.

making up the disk, pressure is initialized with a value $p$ given by the solution to the static problem, $\nabla p=\rho \mathbf{g}$, where $\rho$ is the density. For reference, the analytical solutions for rigid disks are also shown in the figure. When $\mu=0.3$, the disk slides as it rolls. In the case $\mu=0.9$, the point of contact between the disk and the inclined plane does not slip; and, in both cases, the deformable disk moves slower than the comparable rigid disk.

The next test involves the same parameters except that the coefficient of friction is held fixed at $\mu=0.5$ and the angle of inclination is varied, $\theta=\pi / 3$ and $\pi / 6$. Fig. 3 shows the center-of-mass position of the disk as a function of time for each simulation and the corresponding exact solution for a rigid disk. The computed solutions for the deformable disks compare well with the rigid-disk solutions, and as before, the deformable disks move slower than the rigid disks.

The above simulations show reasonable accuracy, relative to the rigid-disk solutions, even on a coarse mesh (side length $0.5 R$ ). Using this mesh size, the largest difference between the rigid-disk solution and the computed, deformable-disk solution occurs when $\mu=0.5$ and $\theta=\pi / 6$ (bottom curve in Fig. 3). The next figure indicates that simulations for this case converge and approach the analytical solution as the mesh is refined. Fig. 4 shows calculations with square computational elements having side lengths $0.5 R, 0.25 R$, and $0.125 R$. With 16 elements across the diameter, the agreement between the analytical solution for a rigid disk and the computation is excellent; and with 8 elements across the diameter the discrepancy is small. In the next section, simulations are performed on a polydisperse sample of grains where the resolution varies from

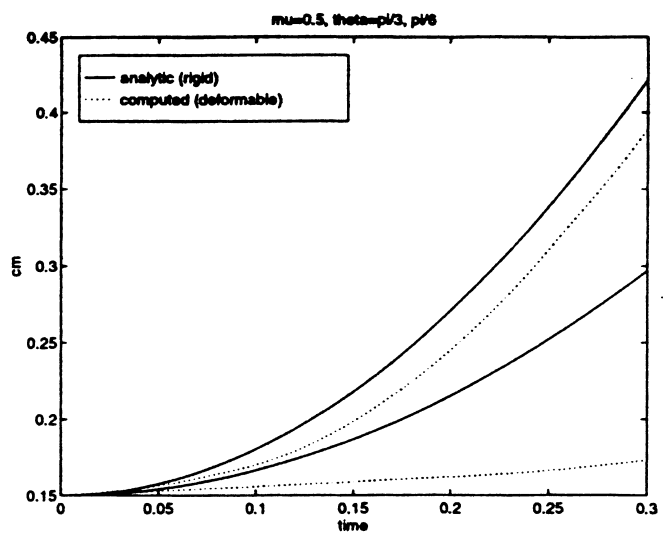

Fig. 3. Center-of-mass position ( $x$-component) for a deformable disk as a function of time (dotted lines) for simulations with friction coefficient $\mu=0.5$ and angles of inclination $\theta=\pi / 3$ and $\pi / 6$. The analytical solution for a rigid disk (solid lines) is also shown for comparison. In both the computed and analytical solutions, the curves for $\theta=\pi / 3$ lie above the curves for $\theta=\pi / 6$. 


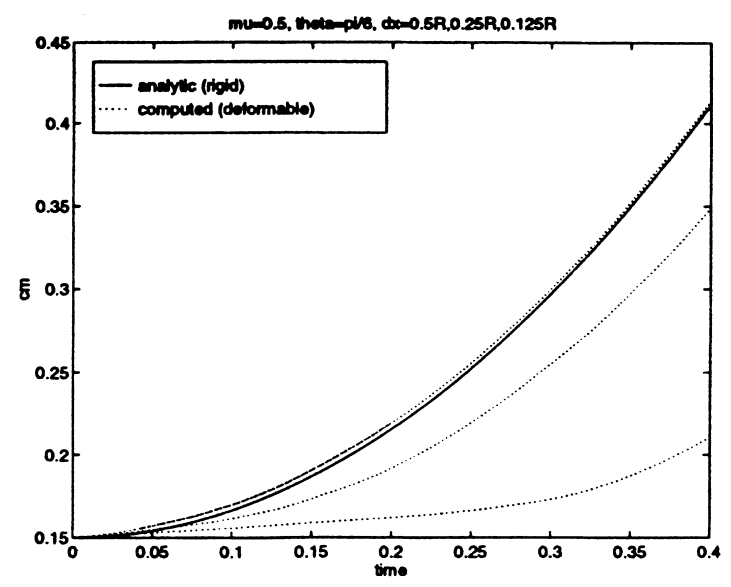

Fig. 4. Center-of-mass position ( $x$-component) for a deformable disk as a function of time (dotted lines) for simulations with friction coefficient $\mu=0.5$, angle of inclination $\theta=\pi / 6$, and meshsizes with side length $0.5 R, 0.25 R$, and $0.125 R$. The analytical solution for a rigid disk (solid lines) is also shown for comparison.

about 4 to 32 elements across the diameter, with the average being about 8 elements. The contact forces should therefore be reasonably resolved, even for the smallest grains.

\subsection{Granular shear simulation}

An example of the kind of problem that can be investigated with this contact algorithm is the shearing of granular material. Granular materials have many interesting properties at least in part attributed to intergranular contact [10]. The simulation of large shear in granular material is a very difficult problem numerically, requiring the inclusion of many grains and the accurate modeling of contact forces and grain deformation. The relative motion of the grains means each grain will encounter many others. Only some of the contacting grains will initially be neighbors, so a priori determination of contacting faces is essentially impossible. Without an efficient contact algorithm the number of grains considered quickly limits the size of problem that can be investigated.

As a demonstration calculation, the shearing of 369 cylindrical grains is simulated. The grain sizes are chosen to be representative of a granular explosive and range in diameter from 31 to $250 \mu \mathrm{m}$ with average $57 \mu \mathrm{m}$. The initial configuration can be seen in Fig. 5, where grains are colored differently only to distinguish them from each other. The black background corresponds to void area . The grains are modeled using Von Mises plasticity with linear hardening and material constants chosen to approximate the energetic crystal HMX. The shear modulus is $7.5 \mathrm{GPa}$, the bulk modulus is $11.4 \mathrm{GPa}$, the yield strength is $0.3 \mathrm{GPa}$, the hardening modulus is $0.01 \mathrm{GPa}$ and the density is $1900 \mathrm{~kg} / \mathrm{m}^{3}$. Shearing is accomplished by fixing the overlapping grains to the left and right boundaries. The left boundary is fixed and the right moved upward at $0.1 \mathrm{~mm} / \mu \mathrm{s}$ resulting in an average shear strain rate of $8 \times 10^{4} / \mathrm{s}$. The initial geometry is constructed periodic in the vertical direction so that material which flows out the top reappears at the bottom and the calculation simulates shear of an infinite periodic slab.

The entire computational domain size is $1.2 \mathrm{~mm}$ square and is discretized with a $150 \times 150$ uniform grid and 4 material points per element. For this discretization, the smallest grains have 4 elements across the diameter. This is clearly insufficient to resolve the stress distribution within the small grains. The objective is to accurately calculate the contact forces and find the load carrying paths in the granular medium. Momentum and energy transport during collisions have been investigated with good results for similar resolutions [2], and the results of the previous section show reasonable accuracy for contact forces. As a demonstration of the numerical technique, this calculation models large material distortion developed by an average shear in excess of $80 \%$. The calculation required approximately $30 \mathrm{~h}$ on a single processor of a Cray YMP, to run 25,124 computational cycles.

The first illustration of the need to correctly handle contact for this system may be seen in Fig. 6. Both figures (a) and (b) are for the same initial configuration (seen in Fig. 5), numerical resolution, and average 


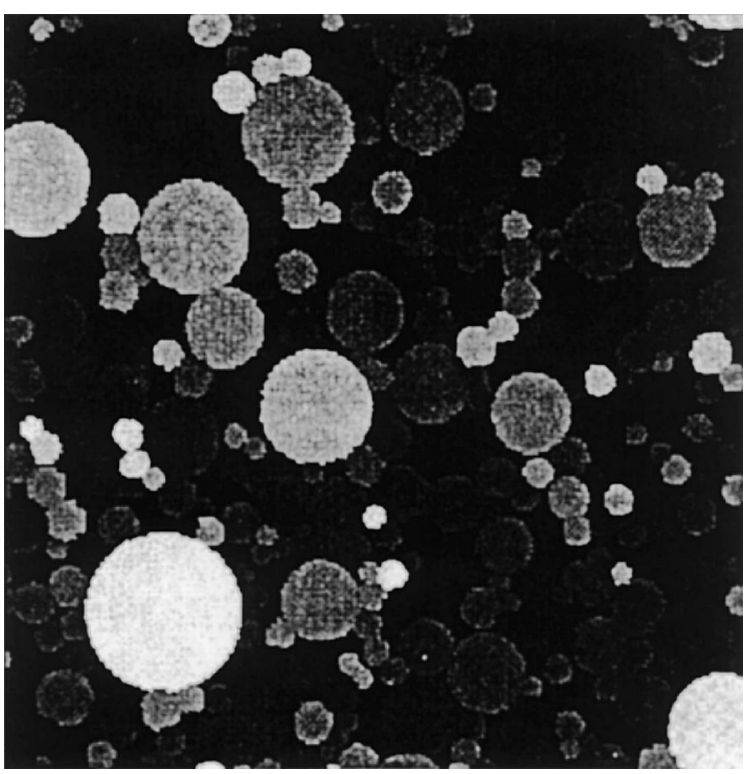

Fig. 5. Initial configuration for granular shear calculation.

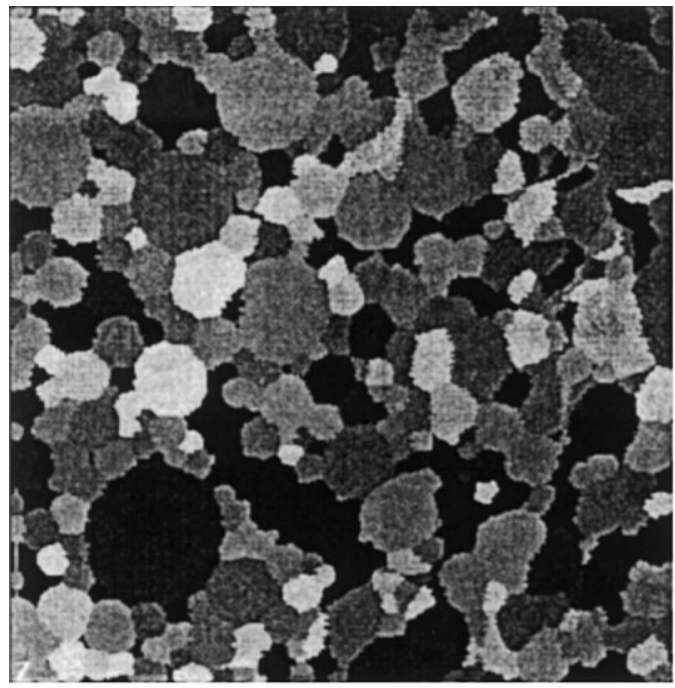

(a)

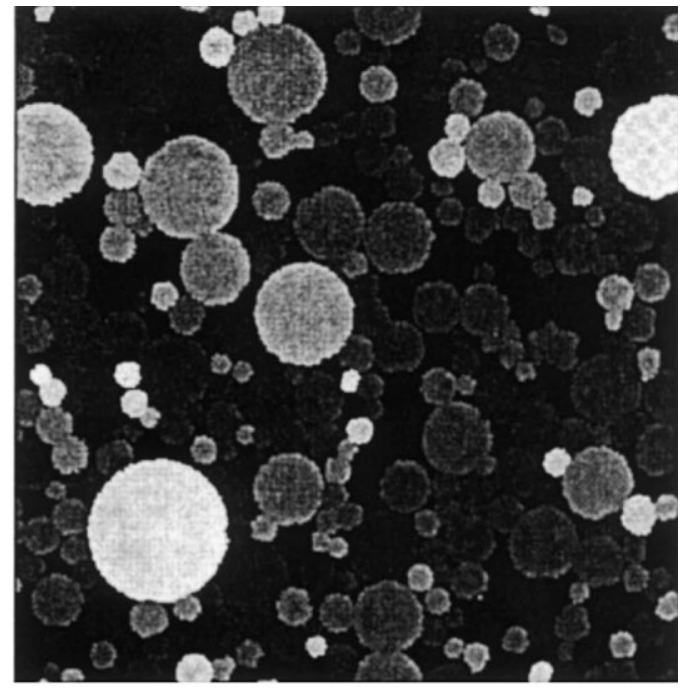

(b)

Fig. 6. Deformation at 75\% shear for cases (a) no slip, and (b) frictional slip, contact conditions.

shear $(75 \%)$. The grain coloring is not the same because the input to the code is processed differently with and without the contact algorithm. Results before implementation of the contact algorithm, where contact conditions are no slip, are depicted in Fig. 6a. The results after implementing the contact algorithm, with friction coefficient $\mu=0.3$, are depicted in Fig. 6b. For the no slip case, there is extensive plastic deformation of the grains and large voids are created. For the case where contact is frictional slip, the situation is completely different. Shear deformation is accommodated by grain rolling and sliding with fairly little plastic deformation. The importance of contact forces in granular material deformation is clear.

Further investigation into the simulation with frictional contact reveals features found experimentally. It has been found that, when loaded, only a small fraction of the grains are significantly stressed. These grains form chains and are the primary load carrying mechanism in granular materials. The phenomenon has been 


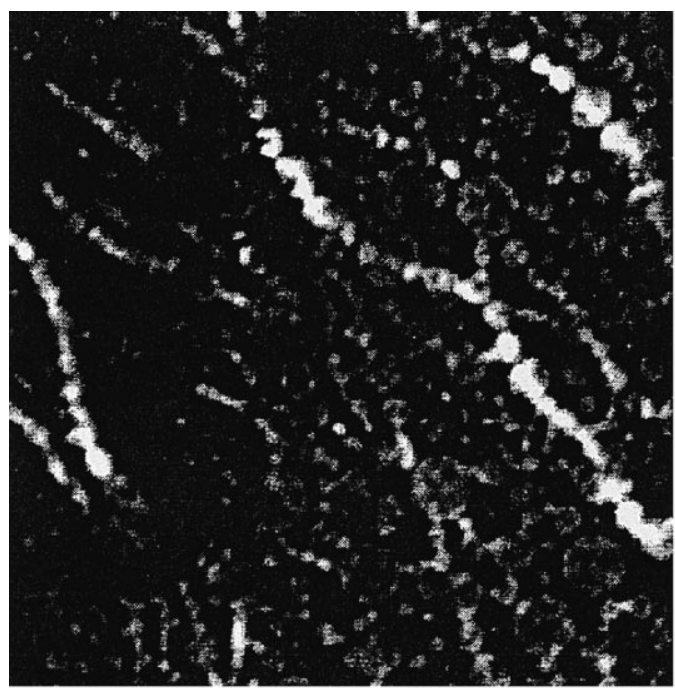

(a)

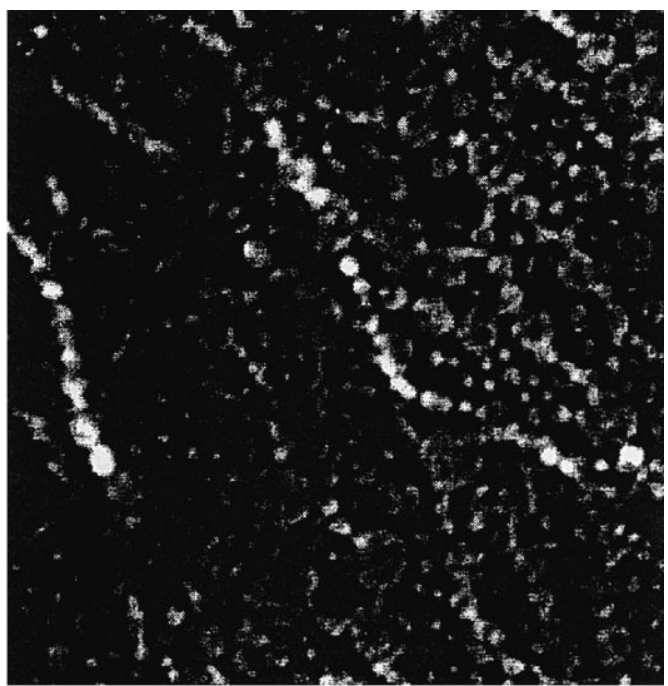

(b)

Fig. 7. Principal stress difference indicating force chains at (a) $63 \%$ and (b) $71 \%$ shear.

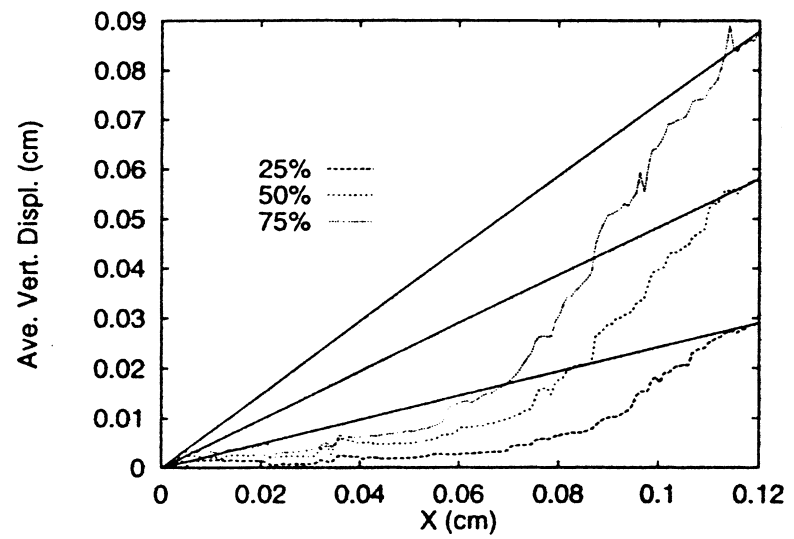

Fig. 8. Average vertical displacement (dashed lines) and uniform displacement (solid lines) at 25\%, 50\% and $75 \%$ shear.

extensively investigated (e.g. [23]) and is known as stress bridging. In the case where granular material is compressed uniaxially the network of force chains is stationary in time over a range of deformation. As demonstrated experimentally [13], shearing is fundamentally different. The confining force during constant volume shearing fluctuates dramatically, and stress bridging networks appear briefly before being replaced by different networks.

Transient stress bridging, or binding, is seen in the numerical simulation with frictional contact. Force chains are created when the grains bind, i.e. translation and sliding are insufficient to accommodate the deformation, and the grains deform. As the shearing proceeds binding is eventually relieved and the force chain disappears. The process is repeated, typically involving different grains in the force chains, as the deformation proceeds. Experimentally stress bridging is observed using photoelasticity, an optical technique which brightens stressed grains with large differences in principal stresses. To produce this effect the difference in principal stress throughout the computational domain is plotted in Fig. 7. Fig. 7a is for 63\% shear and Fig. $7 \mathrm{~b}$ for $71 \%$ shear. The white areas indicate regions of large principal stress difference. Force chains involving many grains are seen in each frame. Note the evolution in the pattern over the $8 \%$ shear between frames. The force chain in the upper right corner disappears, and the force chain nearer a main 
diagonal shifts, involving different grains. The force chain in the bottom left corner persists. Frames much earlier or later in the deformation have much different force chain patterns.

Another characteristic of granular shear is shear banding, or the localization of deformation into a narrow band a few grains wide. Shear banding is also present in the numerical simulation and may be seen by averaging the vertical displacements of all material in each (vertical) column of computational elements. The average vertical displacement is depicted at $25 \%, 50 \%$ and $75 \%$ shear in Fig. 8. Small oscillations in the curves are on account of grain rotations. The straight lines indicate uniform deformation. Motion of the grains is concentrated near the moving boundary on the right. The shear band involves approximately $1 / 3$ of the domain, or is 7 mean particle diameters wide. This value is in good agreement with data for low shear rates (5-15 mean particle diameters) for a variety of materials, for example [14], but somewhat below that for very high shear rates on granular alumina (20-40 mean particle diameters in the absence of fracture) [16].

\section{Conclusions}

An algorithm has been presented for applying a Coulomb friction model to the motion of granular material. The key to the algorithm is not to consider pairwise interactions of grains, but rather to use a common frame in which frictional constraints can be applied for all the grains at once, with one sweep through the computational domain. When incorporated into the material-point method, the result is an algorithm, linear in the number of grains, that is able to account for the interactions between a large number of grains, as well as the internal deformation of each granule.

Shearing of granular material is an example of the kind of problem that can be addressed with this method. To model such a problem correctly requires a large number of grains and the accurate modeling of contact forces and grain deformation. Without an efficient contact algorithm, the problem size is severely limited. As a demonstration calculation, the shearing of 369 , polydisperse grains is simulated. This calculation involves contacting bodies, where the contacting surfaces are not known a priori, and an average shear in excess of $80 \%$ that results in large deformations of the grains. A comparison of a simulation using noslip contact with one using sliding frictional contact shows a dramatic change in the predicted deformation and highlights the need for accurate contact algorithms. The realistic friction model also shows the transient force chains and localized deformation characteristic of experimental observations of sheared granular specimens. The success of this simulation indicates the promise of the method for micro mechanical analyses of granular material.

\section{Acknowledgements}

This work was performed at Los Alamos National Laboratory under contract W-7405-ENG-36 for the US Department of Energy. The work of DS was partially supported by Los Alamos National Laboratory under BOA 0409J0004-3P.

\section{References}

[1] D. Baraff, Issues in computing contact forces for nonpenetrating rigid bodies, Algorithmica 10 (1993) $292-352$.

[2] S.G. Bardenhagen, J.U. Brackbill, D.L. Sulsky, Shear deformationin granular material, in: Proceedings of the 11th International Detonation Symposium, 31 August- 4 September 1998, to appear.

[3] D.J. Benson, A mixture theory for contact in multimaterial Eulerian formulations, Comput. Meth. Appl. Mech. Eng. 97 (1997) $59-86$.

[4] J.U. Brackbill, H.M. Ruppel, FLIP: A method for adaptively zoned particle-in-cell calculations in two dimensions, J. Comput. Phys. 65 (1986) 314-343.

[5] J.U. Brackbill, D.B. Kothe, H.M. Ruppel, FLIP: A low-dissipation, part method for fluid flow, Comput. Phys. Comm. 48 (1988) 25-38.

[6] P.A. Cundall, O.D.L. Strack, A discrete numerical model for granular assemblies, Geotechnique 29 (1979) 47-65.

[7] P.A. Cundall, Formulation of a three-dimensional distinct element method - Part I. A scheme to detect and represent contacts in a system of many polyhedral blocks, Int. J. Rock Rock Mech. Min. Sci. Geomech. Abstr. 25 (1988) 107-116. 
[8] C. DeBoor, A Practical Guide to Splines, Springer, New York, 1978.

[9] H.M. Jaeger, S.R. Nagel, R.P. Behringer, Granular solids liquids and gases, Rev. Modern Phys. 68 (1996) 1259-1273.

[10] H.M. Jaeger, S.R. Nagel, R.P. Behringer, The physics of granular material, Physics Today 49 (1996) 32-38.

[11] C.H. Liu, S.R. Nagel, D.A. Schechter, S.N. Coppersmith, S. Majumdar, O. Narayan, T.A. Witten, Force fluctuations in bead packs, Science 269 (1995) 513-515.

[12] A. Mehta, G.C. Barker, The dynamics of sand, Rep. Progress Phys. 57 (1994) 383-416.

[13] B. Miller, C. O'Hern, R.P. Behringer, Stress fluctuations for continuously sheared granular materials, Phys. Rev. Lett. 77 (1996) 3110-3113.

[14] V.F. Nesterenko, M.A. Meyers, H.C. Chen, Shear localization in high-strain-rate deformation of granular alumina, Acta. Mater. 44 (1996) 2017-2026.

[15] C.E.D. Ouwerkerk, A micromechanical connection between the single-particle strength and the bulk strength of random packings of spherical particles, Powder Technol. 65 (1991) 125-138.

[16] G. Scarpelli, D.M. Wood, Experimental observations of shearband patterns in direct shear tests, in: P.A. Vermeer, H.J. Luger (Eds.), Proceedings of the IUTAM Conference on Deformation and Failure of Granular Materials, Delft, 31 August3 September, 1982, pp. 473-484.

[17] D. Sulsky, J.U. Brackbill, A numerical method for suspension flow, J. Comput. Phys. 96 (1991) 339-368.

[18] D. Sulsky, Z. Chen, H.L. Schreyer, A particle method for history-dependent materials, Comput. Meths. Appl. Mech. Engrg. 118 (1994) 179-196.

[19] D. Sulsky, S.J. Zhou, H.L. Schreyer, Application of a particle-in-cell method to solid mechanics, Comput. Phys. Commun. 87 (1995) 236-252.

[20] D. Sulsky, H.L. Schreyer, A particle method with large rotations applied to the penetration of history-dependent materials, in: Symposium on Advances in Numerical Simulation Techniques for Penetration and Perforation of Solids, The American Society for Mechanical Engineers AMD-Vol. 171, 1993, pp. 95-102.

[21] D. Sulsky, H.L. Schreyer, The particle-in-cell method as a natural impact algorithm, in: Advanced Computational Methods for Material Modeling, The American Society for Mechanical Engineers AMD-Vol. 180, PVP-Vol. 268, 1993, pp. $219-229$.

[22] D. Sulsky, H.L. Schreyer, Axisymmetric form of the material point method with applications to upsetting and Taylor impact problems, Comput. Meths. Appl. Mech. Engrg. 139 (1996) 409-429.

[23] T. Travers, M. Ammi, D. Bideau, A. Gervois, J.C. Messager, J.P. Troadec, Mechanical size effects in 2D granular media, J. Phys. France 49 (1988) 939-948.

[24] R.L. Williamson, Parametric studies of dynamic powder consolidation using a particle-level numerical model, J. Appl. Phys. 68 (1990) 1287-1296. 